GLOBAL JOURNAL OF MEDICAL SCIENCES VOL 10, NO. 1\&2, 2011: 19-27

\title{
CLINICAL NURSING RESEARCH: A TOOL FOR PROFESSIONAL DEVELOPMENT
}

\section{I. AKPABIO}

\begin{abstract}
This paper aimed at highlighting the importance of Clinical Nursing Research (CNR) in the professional development of Nursing. CNR often involve patient-oriented research, epidemiologic and behavioural studies, outcomes and health services research. These are all essential in today's political and societal health care environment with growing shifts and disconnects in patients' care, and the need for availability of human, material and financial resources required in meeting the health care needs. These and many more require the best available evidence, which could be acquired through CNR. However to achieve the benefits of CNR for professional development, Challenges including low quality research by new and inexperienced researchers; non-availability of research findings to nurses in the practice sectors; inability to identify and work on priority areas needed to improve practice; poor collaboration among researchers and inability to integrate research into practice should be addressed. The key to building and sustaining successful programmes in CNR and evidence-based practice could involve the ground work of establishing forums and structures such as research units in health institutions where discussions and activities about the processes for research and evidence-based practice could be promoted.
\end{abstract}

KEYWORDS: Clinical, Nursing, Research, Practice, Structures.

\section{$1.1 \quad$ INTRODUCTION}

Professional development represents the development of Nursing Profession. Nursing profession is all embracing, being very wide in scope. Sometimes, a nurse in his/her clinical practice could be acting as a teacher, Advocate, Counselor, welfare Officer, Team Leader, Coordinator of health programmes to mention but few of the roles.

Furthermore, Nursing when defined in terms of its function has an area of independent professional practice, which could unfortunately be omitted or ineffectively performed when nurses fail to investigate grey areas of the professional practice. Therefore, opportunities for Clinical Nursing Research exist in every contact with a client in the health arena.

In its definition, Clinical Nursing Research could be defined in terms of the contents or in terms of identifying the researcher, the setting for the investigation or the purpose of the study. Defining Clinical Nursing Research in terms of the contents, the National Institutes of Health's Centre for Scientific Website (2011) documented it in three dimensions as follows:

\section{Patient-Oriented Research:}

i.e. Research conducted with human subjects (or on material origin such as tissues, specimens, and cognitive phenomena), for which an investigator (or colleague) directly interacts with human subjects. Excluded from this definition is an in vitro study, which utilizes human, tissues that cannot be linked to a living individual.

Patient-oriented research includes:

(a) Mechanism of human disease,

(b) Therapeutic interventions

(c) Clinical trials or

I. I. Akpabio, Department of Nursing Science, College of Medical Sciences, University of Calabar, Cross River State, Nigeria. 
(d) Development of new technologies

\section{Epidemiologic and behavioural} studies:

Although the term behavioural epidemiology has been used in literature since the late 1970s, it has not been clearly defined. However, according to Sallis, Owen and Fotheringham, (2000), it specifies a systematic sequence of studies on health related behaviours, leading to evidence-based interventions directed at populations. The phases are 1) Establish links between behaviours and health; 2) Develop measures of the behavior; 3) Identify influences on the behavior; 4) Evaluate interventions to change the behavior and 5) Translate research into practice. These are all useful in professional development of nursing.

\section{Outcomes research and health services Research:}

Outcomes research refers to research (usually medically related), which investigates the outcomes of health care practices (Clancy, Eisenberg and John, 1998). Furthermore, it is also defined as the study of the end results of health services that takes patients' experiences, preferences and values into account - is intended to provide scientific evidence relating to decisions made by all who participate in health care.

When defined in terms of the researcher, the setting for the investigation or the purpose of the study, CNR could be presented as a research carried out by nurses in the clinical setting and designed to provide information that could help improve patient care. Other professional staff may also participate in the research (Jacobson, 2004).

A critical analysis of the definitions highlights CNR as patient-oriented study by nurses and other professional staff in the health arena for the purpose of improving patient care and health outcomes. There could therefore, not exist any professional development in Nursing without clinical nursing research.

Professional development involves professional standards, increased accountabilities, effective and efficient acquisition and management of human, material and financial resources. These are all inextricably linked with clinical investigation for empirical facts to guide performance. This paper aims at highlighting the scope and domain, the designs and steps in conducting clinical nursing research, the benefits of clinical nursing research in professional development as well as measures for advancing professional development through clinical nursing research.

\subsection{THE SCOPE AND DOMAINS OF CNR}

Nursing Research aims at generating and assessing evidence for nursing practice. The domain of clinical nursing research represents the specialty practice area for nursing research. The Clinical Nursing Research 2010 Domain of Practice Committee (2009) identified five distinct categories or dimensions of clinical nursing research domain, each housing a cluster of activities. These include the following:

\subsubsection{The clinical practice dimension:}

(i) This dimension is concerned with provision of direct nursing care and support to participants, which could include patients, their families and significant others.

(ii) The approach is with the use of the nursing process.

(iii) Care requirements are determined by the scope of the study, the clinical condition of the patient and clinical effects of research procedures. In the clinical practice dimension, four activities are involved as follows:

CP 1: provision of direct care to research participants (i.e. Interact with research participants to provide nursing care or administration of research interventions or collection of specimen)

CP 2: Providing teaching to research participants and family regarding study participation, participants current clinical condition, and/or disease process.

CP 3: Monitoring the research participants and report potential adverse events to a member of the research team.

CP 4: Recording research data (e.g. documentation of vital signs, participants' responses to care, the medical records' data collection sheet.

\subsubsection{Study Management Dimension}

This involves management of clinical activities or research support in order to ensure patients' safety, address clinical needs and protocol integrity for accurate data collection. According to Clinical Nursing Research 2010 Domains of 
Practice Committee (2009), it includes at least twenty-three activities as follows:

SM 1: Participating in study development

SM 2: Participating in research participants' recruitment

SM 3: Participating in screening potential research participants for eligibility

SM 4: Coordinate and facilitate the collection of research specimens

SM 5: Developing study specific materials for research participants' education

SM 6: Performing quality assurance activities to ensure data integrity.

SM 7: Participating in the preparation of reports for appropriate regulatory and monitoring bodies/Boards

SM 8: Facilitating accurate communication among research sites

SM 9: Facilitating communication within the research team

SM 10: Contribute to the development of case report forms

SM 11: Participating in the set up of a study specific data base

SM 12: Complying with International Conference on Harmonization of Good Clinical Practice guidelines

SM 13: Collecting data on research participants, based on study endpoints.

SM 14: Facilitating scheduling and coordination of study procedures

SM 15: Providing nursing expertise to the research team during study development and implementation

SM 16: Protecting research participants' data in accordance with regulatory requirements.

SM 17: Participating in site visits and/or audits.

SM 18: Supporting study grants and budget development

SM 19: Overseeing human resources (people) related to research process

SM 20: Recording of data on approved study documents (e.g. case report forms, research/study papers.

SM 21: Facilitating, processing and handling (Storage and Shipping) of research specimens.

SM 22: Identifying implications clinical during development care study staff competencies, equipments).

SM 23: Participating in the identification and reporting of research trends.

\subsubsection{Care Coordination and Continuity Dimension}

This domain involves coordination of research and clinical activities to meet clinical needs of patients; complete study requirements and manage linkages with referring and primary care providers. Care coordination and continuity dimension covers series of ten activities as follows:

CCC 1: Facilitating the education of the interdisciplinary team on study requirements.

ccc 2: Collaborating with the interdisciplinary team to create and communicate a plan of care that allows for safe and effective collection of clinical research data.

CCC 3: Coordinating research participant study visits.

CCC 4: Providing nursing leadership within the interdisciplinary team.

CCC 5: Coordinating interdisciplinary meetings and activities in the context of a study

CCC 6: Coordinating referrals to appropriate interdisciplinary services outside the immediate research team

CCC 7: Communicating the impact study procedures on the research participants.

CCC 8: Providing nursing expertise to community based health care personnel related to study participation.

CCC 9: Facilitating research participants enquiries and concerns

CCC 10:Providing indirect nursing care (e.g. participation in clinical, unit and/or protocol rounds. Scheduling study related tests in the context of research participation.

\subsubsection{Human Subjects Protection dimension} This aspect of clinical nursing research has to do with facilitation of informed participation by diverse participants in clinical research. The human subject protection domain should cover at 
least six activities as follows:

HSP 1: Facilitating the initial and ongoing informed consent.

HSP 2: Supporting research participant in defining his/her reasons and goals for participating in a study.

HSP 3: Collaborating with the interdisciplinary team to address ethical conflicts.

HSP 4: Coordinating research activities to minimize subject risk

HSP 5: Serving as Interdisciplinary Board member.

HSP 6: Managing potential ethical and financial conflicts of interest for self.

\subsubsection{Contributing to Clinical Science}

This aspect involves contribution as a research team member to the development of new ideas from the study, explorations of innovations arising from clinical research findings to guide practice. This domain covers, at least nine distinct activities including the following:

CS 1: Disseminating clinical expertise and best practices related to clinical research through presentations, publications and/or interactions with nursing colleagues.

CS 2: Serving as an expert in a specialty area (E.g. grant reviewer, Editorial Board, Presenter.

CS 3: Participating in the query and analysis of research data.

CS 4: Generate practice questions as a result of a new study procedure or intervention.

CS 5: Collaborating with the interdisciplinary team to develop innovations in care delivery that have the potential to improve patient outcomes and accuracy of data collection.

CS 6: Identifying questions appropriate for clinical nursing research as a result of study team participation.

CS 7: Mentoring junior staff and students participating as members of the research team.
CS 8: Performing secondary data analysis to contribute to the development of new ideas.

CS 9: Serving as a resource to new investigator(s).

In summary therefore, clinical research nurses have a central role in assuming participant safety, ongoing maintenance of informed consent, integrity of protocol implementation and accuracy of data collection, data recording and follow-up. Furthermore, clinical nursing research can never be conducted by nurse educators alone in isolation from clinical nurse practitioners.

\subsection{Significance of the various activities:}

The activities listed under the domains/dimensions have great significance in achieving professional development of nursing. They are skills needed for qualitative and quantitative research, which similarly play vital roles in developing nursing knowledge. The activities form realistic strategies in the conduct of research. For instance, valid knowledge from research requires accurate selection of sample with correct sample size and sampling technique; accuracy in data collection and analyses of data; and unbiased presentation of new ideas for implementation.

\subsection{Clinical nursing research designs}

There are various designs that could be adopted in clinical Nursing Research. However, the types of questions to be answered often determine the best research design to use (Martin and David, 2007). Using the principle that the level of knowledge available on a research topic determines the type of design that could be used to study the topic, for instance the four most common types of questions in clinical nursing research are therapy, diagnosis, etiology and prognosis, a randomized controlled trial would not be feasible for a prognosis study while the best design for prognosis should be a cohort study.

Brink and Wood (2010) presented three levels of advanced designs in Nursing Research to include: Theoretically based experimental designs; comparative and correlated survey designs; and exploratory-descriptive research designs. The study design types for various clinical questions could be as follows: 


\begin{tabular}{|l|l|}
\hline Clinical Question & Suggested Research Design \\
\hline Therapy & $\begin{array}{l}\text { Randomized control trial (RCT) is preferable otherwise; cohort } \\
\text { study, case control study, case series study. }\end{array}$ \\
\hline Diagnosis & Prospective, blind comparison to a reference standard. \\
\hline Aetiology or Harm & Cohort study, case control study, case series. \\
\hline Prognosis & $\begin{array}{l}\text { Cohort study is preferable, otherwise: case control study, case } \\
\text { series. }\end{array}$ \\
\hline Quantity improvement & RCT and qualitative studies. \\
\hline Cost & Economic evaluation design \\
\hline
\end{tabular}

Although most clinical research in nursing focus on quantitative designs, qualitative research designs similarly play an important role in clinical nursing studies. How qualitative research is described varies in different fields, but an over aching theme is that qualitative research is research focusing on how individuals (or groups) view and understand their world and experiences. Greenhaigh and Tailor (2007) identity two points of qualitative research in health sciences as "aimed to make sense of, or interpret phenomena in terms of the meanings people bring to them" and "research that defines preliminary questions, which can then be addressed in quantitative studies". In Nursing, qualitative research designs are best for understanding the meaning of illness or patient experiences, attitudes and beliefs. The short coming in most qualitative studies could be the inability of lending accurate and valid results or evidence to guide clinical practice as the case may be in systematic empirical studies of some quantitative research.

\subsection{GUIDES IN CONDUCTING CLINICAL NURSING RESEARCH}

Researchers who wish to conduct clinical nursing research must identify a problem that is of clinical importance. The problems that are encountered everyday in the health care arena provide the basis for these research problems and questions. Observations that are made could translate into possible research problems to be approached, especially in situations that produce negative outcomes. Resources and expertise that are discovered through research are then shared with other health care providers.

To conduct clinical nursing research, the researcher must have knowledge of research designs and methods and while using any of the designs, should identify and ensure that strengths and weaknesses of each design in sampling methods, reliability, validity, data analysis and issues related to human subjects are addressed. It is necessary to comprehend the analytical processes necessary for successful research. It may also be needed to negotiate funding for research projects and ensure that the research is being conducted in accordance with ethical guidelines. The key skills that aid in successful clinical nursing research are: communication, management, analytical, organizational as well as clinical knowledge (Hastings, 2009). Furthermore the clinical nurse researcher should be an advanced practice nurse who is academically prepared with critical thinking ability.

The steps in clinical Nursing research largely depend on whether qualitative or quantitative study design is used.

\section{A. Qualitative design}

For a qualitative design (non-linear) the steps according to Polit (2011) should include the following:

(i) Identification of research problem

(ii) Literature review

(iii) Selecting and gaining entry into research sites.

(iv) Utilizing a design that emerges as data is collected e.g. ethnographic, phenomenological, grounded theory, historical research designs.

(v) Addressing ethical issues

(vi) Collecting the data

(vii) Analyzing the data

(viii) Interpretation of the research findings

(viii) Communicating the study findings

(ix) Participating in the process of disseminating the findings from the research.

\subsubsection{Usefulness of some qualitative designs: \\ Qualitative designs could include Phenomenological and Ethnographic designs.}

a) Usefulness of Phenomenological design: With this, the inquiry just like in any qualitative study is carried out under naturalistic setting so 
that the context is considered part of the phenomenon, but the researcher makes no attempt to place experimental controls on the phenomenon being studied (Morse and Field, 2005). The numbers of participants are usually small but large enough to provide rich data. According to Morse and Field (2005), Phenomenology is a qualitative study in which researchers' attempt to discover the meaning of lived experiences by human beings as they exist in the world. It could involve six activities that include turning to the phenomenon; investigating experiences as human beings live them; reflecting on the essential themes; using art and writing to discuss the phenomenon; and balancing the research context (Van-Manen, 2007). Spiegelberg (2002) believed that Phenomenology begins in silence while observing what is happening around the individual. Phenomenological study could therefore be part of research as nurse clinicians practice in their natural settings in the health care sector.

\section{b) Usefulness of Ethnographic design:}

Ethnography involves studying a group of people in their own environment. The goal of ethnography is to learn from, rather than about, people from various cultures, religions, and ethnic groups (Spradley, 2002). There is a concern with the meaning and actions of the people being studied, resulting in the description and interpretation of cultural patterns. The researcher takes into account the participant's point of view. Researcher becomes the data collection instrument through observation and recording of data. Similarly, ethnographic study could be part of research as nurse clinicians practice in their natural settings in the health sector.

\section{B. Quantitative design}

In Quantitative designs (linear), the steps could be as follows:

- Identification of the research problem

- Review of related literature

- Framing the problem conceptually

- Formulating hypotheses

- Selecting a design

- Identifying population and sampling plan

- Selecting and testing methods to measure variables

- Protecting human rights

- Reviewing and finalizing the research plan

- Collecting the data
- $\quad$ Analyzing the data

- Interpretation of the study findings

- Communicating the research findings

- Participating in the process of disseminating the findings

Thus, looking at the steps, it could be observed that Qualitative designs are more flexible than the quantitative designs while the quantitative designs involve statistical analysis to enhance the provision of reliable results of the relationship among the variables that are being studied.

\subsection{BENEFITS OF CNR}

The need for research in practice is well documented within nursing and other health care disciplines. This argument is predicated on the belief that clinically applied research will inform and improve practice and health service delivery resulting in better outcomes for consumers and their families.

As new technologies are constantly being introduced, new clinical problems that result in research questions are being raised, which all require clinical nursing research to resolve. In this way, it improves clinical expertise and personal knowledge, helps to implement changes and provide excellence in nursing care. By so doing, clinical nursing research could forge a professional legacy by promoting lifelong professional development of the discipline of nursing and supports the fact that nursing is a professional discipline with specified areas for independent practice. For instance, how do organizations and nurse leaders recruit and retain the best practical nurses available in the midst of profound numbers of varied training institutions and programmes? How do they bring new employees into greater levels of professional competency, proficiency and expertise to achieve the best possible patient care outcomes in the least amount of time with the fewest possible resources? How do they manage escalating of technologies for a global advance? These all require the best available evidence to guide performance.

American Nurses' Association (2004) opined that nurses are expected to recognize and respond to a plethora of new demands arising from an ever changing and increasingly complex health care system, multiple new regulations and accreditation processes. Professional standards, increased accountabilities, and financial priorities require more of direct care nurses and nurse leaders that are able to gain knowledge through 
systematic studies and sustain competencies in evolving arenas of practice for quality and safe patient care.

Today's political and societal health care environments include growing shifts and disconnects in patient care needs and the availability of human, material and financial resources required to meet the needs. Nursing professional development specialists are the key to successful succession planning, managing competing priorities, and effecting cost avoidance. These practitioners are more than educators. They could be advanced practice clinical nurses/leaders able to emphasize safety, quality, efficiency and effectiveness of practice; they should be able to rapidly transit diverse generations of nurses into clinical experts. These and many more require the best available evidence, which could be acquired through clinical nursing research.

\subsection{CHALLENGES}

Challenges in utilizing clinical research findings could include: low quality research from new and inexperienced researchers; nonavailability of research findings to nurses in the practice sector. Inability to identify and work on priority areas needed to improve practice and inability to integrate research findings into practice.

Currently, in Nigeria, clinical nursing research is not integrated into everyday duties of the nurse, and there is a need for more trained, clinical nursing researchers. According to Akpabio (2010), most of the research works are carried out in institutions of learning based on only the interest of the researchers and the need to fulfill academic requirement. It is essential for the professionals that are in contact with patients to be active in the research aimed at improving practice. If that is done, they could easily identify the most relevant and important research problems, assist in the research process and show interest in the implementation of the study findings. It is a misconception that clinical nursing research is only conducted by Physicians, graduate students or nurses with doctorate degree.

Similarly, in many instances, problems emanate due to the large gap that is existing between theory and research among nurses. Many nurses due to dearth of post-graduate departments offering higher degrees in nursing cross over to other non-nursing faculties and departments to offer courses but however conduct nursing related research that are mostly outside the wards practice, believing that such studies are still very relevant to the discipline of nursing. Be that as it may, it is documented that research without theory results in discreet information or data which does not add to the accumulated knowledge of the discipline (Kaiser, 2009). According to the author, theory guides the research process, forms the research questions, aids in design selection, analysis and interpretation of findings. It enables the scientists to weave the facts together thereby making the relationship between study findings and practice direct and positive.

Furthermore, Akpabio and Ebong (2010) identified challenges to evidence-based practice under four broad domains including poor accessibility to research findings and insufficient new evidence in the relevant areas of practice; poor organizational support to conduct and use research findings; poor support from significant persons in the health sector and fear of anticipated outcome in using research findings. Each of these four problems areas have in its domain specific challenges.

Another vital challenge in the conduct of clinical nursing research is that CNR involves patients who may be critically ill or sometimes having psychological problems, thus making research for such clients difficult to conduct (Brink and Wood, 2010). Furthermore, the hectic environment of the health care setting and the problem of conducting clinical research on children could all add to already existing myriads of challenges. Additionally, clinical research is increasingly encumbered by high cost and lack of funding, regulatory burdens, and incompatible databases, shortage of qualified investigators, lack of facilities including computers/internet services, and oftentimes lack of willing participants. However, because of the numerous benefits that could be achieved from CNR, efforts at surmounting the challenges should be worthwhile activities.

\subsection{MEASURES OF ADVANCING PROFESSIONAL DEVELOPMENT THROUGH CNR}

Commenting on measures of translating Biomedical research to the bedside, National Institutes of Health's Centre for Scientific Website (2011) opined that clinical nurse researchers should be aware and acknowledge problems or challenges that could hinder the conduct of research in the planning stage so as to minimize 
the negative influence such challenges could have on implementation of the study findings.

The measures of advancing professional development through CNR could be discussed, using the linear framework of structure; process and outcome (Donabedian, 1988). The key to building and sustaining successful programmes in clinical nursing research and evidence-based practice is to lay the ground work by establishing structures and forums where discussions and activities associated with the processes for research and evidence-based practice could occur. Although no one structure could meet the needs of all nursing institutions, several structures should be considered. For instance, Evidence-based practice units could assist nurses in solving clinical practice issues in their units in addition to mentorship programme. Such structures could further promote collaboration with professional colleagues in the area of clinical research.

Furthermore, structures to support research must engage frontline clinicians. Nurses in positions of leadership should exhibit positive attitudes towards clinical research by encouraging involvement in research activities. Such executive actions from the leaders could send messages to other staff that patient care should be based on the best available evidence and should not vary illogically from practitioner to practitioner.

Referring to the process, practice documents such as policies, procedures and guidelines must be written to reflect practices that are based on research and other levels of evidence. To improve research utilization, the focus of research should include the resolution of day-to-day nursing or health problems to enhance clients care. Thus, research problems should originate from the practice arena and attract collaboration between nurses in all sectors, including the clinical areas, administration, industrial and educational sectors. This attempt will not only improve the quality of the research work, but could also improve understanding of research reports by all participants, subsequently resulting in ease of results implementation. This argument is very vital since in many instances the nurse researchers in the education sectors may not be in a position to implement the research findings. On the other hand, where research work originates from the researchers in the education sectors, every effort should be made to include in the research reports, recommendations relating to research implementation, and copies of such research reports should similarly be directed to the relevant practice areas for implementation.

In terms of outcome, the benefits of clinical research can never be obtained if research findings are not implemented or utilized to guide practice. Utilizing nursing research implies using research findings in the development of policies, procedures and standards of practice in patient care, and incorporating research as basis for learning (Akpabio, 2008a). According to the author, if knowledge is not utilized to improve practice, then there is limited or no use to have conducted the research.

\subsection{SUMMARY AND CONCLUSION}

CNR is slowly progressing to an advanced level in recent years to meet the political and health care needs of the society. Progress is limited by insufficient human, material and financial resources required to meet research demands. Development of professional nursing is to a very great extent based on the sciences of nursing, technology, research and evidence-based-practice; practice-basedevidence (i.e. documentation of relevant experiences acquired in the course of clients' care), positive changes whenever necessary and information communication technology. These research oriented professional development roles are necessary for the advancement of present and future professional nursing. Clinical nursing research should cover all the health arenas with increasing emphasis on areas where patients/clients are directly cared for. For this reason therefore, nurse leaders including those involved in direct patients' care in the clinical areas should support nursing research and evidence-based-practice by establishing and sustaining practice-based-evidence, which could further be studied empirically. They could increasingly act as expert change agents and quality improvement specialists to bring about nursing professional development. This recommendation requires closing the gaps in the conduct of research at institutions of learning and research utilization at clinical settings that are observed to exist between nurse educators in institutions of higher learning and nurse leaders in the clinical sectors (Akpabio, 2010).

Opportunities should therefore be created for nurse educators to participate in the clinical areas as consultants while their counterparts in the clinical areas should acquire advanced knowledge and carefully document 
experiences acquired in their specific specialty areas during the performance of duty or services to clients. By so doing, they could also become clinical nurse consultants in their respective areas or domains of care.

\section{REFERENCES}

Akpabio, I. I., 2010. Improving care through nursing research, Hamdard Medicus, Quarterly Journal of Science and Medicine, 53, (4): 18-87.

Akpabio, I. I and Ebong, F. S., 2010. Research Methodology and Statistics in Health and Behavioural Sciences, Calabar: Eclait Biz.

American Nurses' Association., 2009. Committee on nursing practice: Standards of clinical nursing practice, USA: ANA

Brink, P. J and Wood, M. J., 2010. Advanced Design in Nursing Research, available at: http://www. doi.wiley.com.

Clancy, C. M., Eisenberg and John, M., 1998. Outcomes research: Measuring the end result of health care, Science 282, (5387): 245-246.

Clinical Nursing Research 2010 Domains of Practice Committee., 2009. Building the foundations for clinical research nursing: Domains of practice for the specialty area of clinical research nursing, National Institute of Health Clinical Centre, Nursing and patients' care services, available at:http://www.cc.nih.gov/nursing lcrn/DOP document.pdf.

Greenhaigh, P and Tailor, F., 2007. Issues and Challenges in clinical nursing research, available at:http://www.cc.nih.nursing/crn /designs.

Hastings, F., 2009. Specialty areas in clinical research nursing, available at: http://www.cc.nih.gov/nursing.CRN/DOP. document.pdf.
Jacobson, S. F., 2004. Instruments for Clinical Health Care Research, available at: http://www. books.google.com

Kaiser, P., 2009. Nursing Research and Theories, available at: http://www. Currentnursing.com/nursingtheory/r.

Martin, C. R and David, R. 2007. Designs and analyses of clinical nursing research studies, At: http://www.amazon.com

Morse, J and Field, P., 2005. Qualitative research methods for health professionals, Thousand Oaks, CA: Sage Publications

National Institute of Health's Centre for Scientific

Website., 2011. Clinical research nursing domains' of practice, available at: http://www.cc.nih.gov/nursing/crn/DOP d ocument.Pdf.

Polit, D. F., 2008. Nursing research: Generating and assessing evidence for nursing. USA: Centre for Clinical Practice Innovation.

Spiegelberg, H., 2002. The phenomenological movement: A historical perspective, Netherlands: Nijhoff Publishers

Spradley, J. P., 2006. Participant observation, Journal of School Nursing, 22, 212.

Van-Manen, M., 2007. Researching the lived experience, Human science for an action sensitive pedagogy, London: The Althouge Press. 